\title{
Brennecke, Hanns Christof, Niefanger, Dirk, Schnabel, Werner Wilhelm, Akademie und Universität Altdorf, Studien zur Hochschulgeschichte Nürnbergs
}

Boris Klein

\section{OpenEdition}

Édition électronique

URL : http://journals.openedition.org/ifha/6489

DOI : $10.4000 /$ ifha.6489

ISSN : 2198-8943

Éditeur

IFRA - Institut franco-allemand (sciences historiques et sociales)

\section{Référence électronique}

Boris Klein, «Brennecke, Hanns Christof, Niefanger, Dirk, Schnabel, Werner Wilhelm, Akademie und Universität Altdorf, Studien zur Hochschulgeschichte Nürnbergs », Revue de l'IFHA [En ligne], Date de recension, mis en ligne le 01 janvier 2012, consulté le 22 septembre 2020. URL : http:// journals.openedition.org/ifha/6489; DOI : https://doi.org/10.4000/ifha.6489

Ce document a été généré automatiquement le 22 septembre 2020.

(CIFHA 


\title{
Brennecke, Hanns Christof, Niefanger, Dirk, Schnabel, Werner Wilhelm, Akademie und Universität Altdorf, Studien zur Hochschulgeschichte Nürnbergs
}

\author{
Boris Klein
}

C'est en 1504 que la petite cité d'Altdorf tomba sous la domination de Nuremberg, alors une des plus puissantes villes libres de l'Empire. Quelques décennies plus tard, la nouvelle conquête était désignée pour abriter une école secondaire, laquelle fut ensuite élevée au rang d'université en deux temps, entre 1578 et 1622 . Dès lors, la cité indépendante de Nuremberg put se targuer pendant plus de deux siècles de posséder sa propre université, jusqu'à ce que, comme d'autres, l'établissement soit victime des brutales opérations de réorganisation du paysage universitaire germanique au début du XIXe siècle, et qu'il ferme définitivement ses portes en 1809. À l'occasion du bicentenaire de cette disparition, un important colloque a été organisé, dont les actes viennent d'être publiés. Au fil des dix-neuf contributions que contient l'ouvrage, les différents auteurs tentent de faire revivre le riche passé d'Altdorf en multipliant les approches. Après une introduction générale et un premier rappel sur les conditions de naissance de l'université et ses rapports avec le pouvoir politique, l'article de W. Mährle replace les premières décennies d'existence de l'institution dans le contexte de l'humanisme tardif. L'influence et le rayonnement de l'établissement, notamment dans les régions orientales de l'Empire, sont ensuite soulignés par deux études sur les étudiants originaires de Bohême et de Pologne (M. Holy et Z. Pietrzyk). Plusieurs contributions se penchent sur l'enseignement dans les différentes facultés, analysant l'influence de l'aristotélisme (W. Sparn), l'impact des débats théologiques (H.C. Brennecke), la réception de Machiavel (c. Zwierlein), ou encore l'originalité de l'enseignement de la médecine (M.M. Ruisinger) ou la place des réflexions sur le judaïsme et l'islam (H. Bobzin). Au sein de cette série d'articles, on notera l'originale 
contribution de J. Bruning, lequel s'attache à comparer l'Academia Norica avec sa jumelle Helmstedt, autre université luthérienne du nord de l'Allemagne, elle aussi fondée à la fin du XVIe siècle, déclinante à l'époque des Lumières, puis finalement fermée au début du XIXe siècle. Moins bien représentées, l'histoire sociale et l'histoire des rites et cérémonies propres à l'institution font cependant l'objet de deux contributions, l'une de D. Niefanger et de W.W. Schnabel sur les cercles littéraires, et l'autre, particulièrement originale, de H. Maué qui étudie les médailles frappées par l'université à l'occasion des grands événements, ou offertes comme récompense. Enfin, $\mathrm{H}$. Heller rappelle comment les bâtiments de l'ancienne université demeurèrent jusque dans les années 1920 un lieu de formation pour les futurs maîtres d'écoles de Franconie. Particulièrement dense et bien illustré, l'ouvrage a ainsi le mérite d'aborder de nombreux thèmes, ouvrant autant de possibilités à de futures études comparatives. Surtout, il remplit parfaitement son objectif en soulignant la place importante de l'université d'Altdorf dans l'histoire des institutions universitaires à l'époque moderne. Trop souvent négligée, la vie de ces petites universités protestantes défuntes constitue en effet une clef pour mieux comprendre l'émergence des nouveaux modèles universitaires à l'époque des Lumières et au XIXe siècle. Dans cet esprit, le présent ouvrage s'impose comme une référence.

Boris Klein (université Lyon II) 